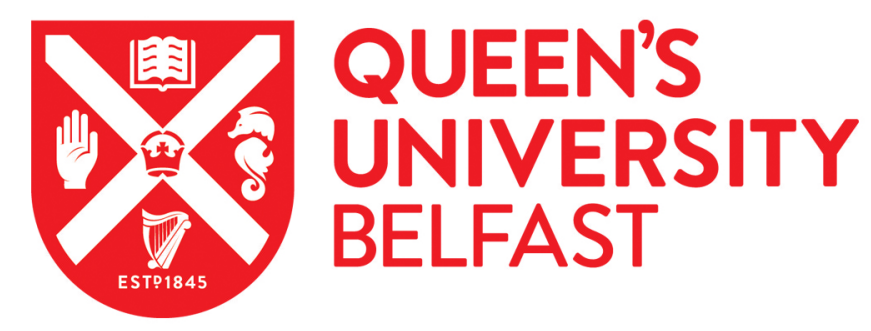

\title{
Reducing potentially inappropriate prescribing for older people in primary care: Cost effectiveness of the OPTI-SCRIPT intervention.
}

Gillespie, P., Clyne, B., Raymakers, A., Fahey, T., Hughes, C., \& Smith, S. (2017). Reducing potentially inappropriate prescribing for older people in primary care: Cost effectiveness of the OPTI-SCRIPT intervention. International Journal of Technology Assessment in Health care, 33(4), 494-503. https://doi.org/10.1017/S0266462317000782

Published in:

International Journal of Technology Assessment in Health care

Document Version:

Peer reviewed version

Queen's University Belfast - Research Portal:

Link to publication record in Queen's University Belfast Research Portal

Publisher rights

(C) Cambridge University Press 2017. This work is made available online in accordance with the publisher's policies. Please refer to any applicable terms of use of the publisher.

\section{General rights}

Copyright for the publications made accessible via the Queen's University Belfast Research Portal is retained by the author(s) and / or other copyright owners and it is a condition of accessing these publications that users recognise and abide by the legal requirements associated with these rights.

Take down policy

The Research Portal is Queen's institutional repository that provides access to Queen's research output. Every effort has been made to ensure that content in the Research Portal does not infringe any person's rights, or applicable UK laws. If you discover content in the Research Portal that you believe breaches copyright or violates any law, please contact openaccess@qub.ac.uk. 
Title:

Reducing potentially inappropriate prescribing for older people in primary care: Cost effectiveness of the OPTI-SCRIPT intervention

\section{Short Title:}

The OPTI-SCRIPT Study: Economic Evaluation

\section{Authors:}

Paddy Gillespie ${ }^{1}, \mathrm{PhD}$, Barbara Clyne ${ }^{2}$, PhD, Adam Raymakers, ${ }^{3}$ MSc Tom Fahey ${ }^{2}$ MD, Carmel Hughes, ${ }^{4} \mathrm{PhD}$, Susan M Smith ${ }^{2}$, MD, on behalf of the OPTI-SCRIPT study team

1 Health Economics and Policy Analysis Centre, National University of Ireland, Galway

2 HRB Centre for Primary Care Research, Department of General Practice, Royal College of Surgeons Ireland Medical School, Dublin

3 Centre for Research on Medical Devices (CURAM), National University of Ireland, Galway 4 School of Pharmacy, Queens University Belfast

\section{Corresponding Author:}

Dr Paddy Gillespie

Address: School of Business and Economics, Institute of Lifecourse and Society (ILAS) Building, National University of Ireland (NUI) Galway, Galway, Ireland.

Email: paddy.gillespie@nuigalway.ie

Phone: $+353(0) 91492501$ or $353(0) 876421488 \quad$ Fax: $+353(0) 91524130$

\section{Support:}

This study is independent research, funded by the Health Research Board (HRB) $\mathrm{PhD}$ Scholars Programme in Health Services Research under Grant No. PHD/2007/16 and the HRB Centre for Primary Care Research under Grant no HRC/2007/1.

We thank all the GP practices and patients who participated in this study. 


\section{ABSTRACT}

\section{Objective:}

This study examines the cost effectiveness of the OPTI-SCRIPT intervention on potentially inappropriate prescribing in primary care.

\section{Methods:}

Economic evaluation, employing incremental cost effectiveness and cost utility analyses, conducted alongside a cluster randomised controlled trial of 21 general practices and 196 patients, to compare a multifaceted intervention to usual practice in primary care in Ireland. Potentially inappropriate prescriptions (PIPs) were determined by a pharmacist. Incremental costs, PIPs, and quality adjusted life years (QALYs) at 12 months follow up were estimated using multilevel regression. Uncertainty was explored using cost effectiveness acceptability curves.

\section{Results:}

The intervention was associated with a non-significant mean cost increase of $€ 407$ (95\% CIs: $-357,1170)$, a significant mean reduction in PIPs of 0.379 (95\% CI: $0.092,0.666)$, and a nonsignificant mean increase in QALYs of 0.013 (95\% CIs: -0.016, 0.042). The incremental cost per PIP avoided was $€ 1,269$ (95\% CI: -1400, 6302) and the incremental cost per QALY gained was $€ 30,535$ (95\% CI: $-334,846,289,498)$. The probability of the intervention being cost effective was 0.602 at a threshold value of $€ 45,000$ per QALY gained and was at least 0.845 at threshold values of $€ 2,500$ per PIP avoided and higher.

\section{Conclusions:}

While the OPTI-SCRIPT intervention was effective in reducing potentially inappropriate prescribing in primary care in Ireland, our findings highlight the uncertainty with respect to its cost effectiveness. Further studies are required to explore the health and economic implications of interventions targeting potentially inappropriate prescribing. 


\section{KEY WORDS:}

Cost effectiveness; Randomised controlled trial, Potentially inappropriate prescribing, Primary care

TRIAL REGISTRATION:

Current controlled trials ISRCTN41694007 


\section{INTRODUCTION}

Potentially inappropriate prescribing describes suboptimal prescribing practices that increase the risk of adverse drug events for patients in circumstances where safer, more effective treatment alternatives are available for the condition of interest (1). Within the overall patient population, older people, with their relatively high prevalence of multimorbidity and consequent polypharmacy, are particularly vulnerable to potentially inappropriate prescribing and pose particularly challenging prescribing decisions for healthcare providers (2). A number of published tools to assess potentially inappropriate prescribing in older people exist, the majority of which are explicit: for example, by listing drugs that should to be avoided (1). Applying these tools, potentially inappropriate prescribing in older people has been found to be common across healthcare settings and can result in increased morbidity, adverse drug events (ADEs), hospitalisations (2,3) and healthcare costs (4). In the Irish healthcare setting, potentially inappropriate prescribing in community-dwelling older Irish people has been shown to be associated with increased ADEs, accident and emergency (A\&E) visits, and poorer health related quality of life (5). Moreover, a recent study estimated that $36 \%$ of those aged 70 years and over had at least one potentially inappropriate prescription (PIP), resulting in additional healthcare expenditures of $€ 45$ million, or $9 \%$ of overall pharmaceutical expenditures for those aged 70 years and over in Ireland (4).

Within this context, the development of healthcare interventions which target reductions in potentially inappropriate prescribing represent an important public health measure, both in terms of their ability to improve patient health outcomes and to reduce the economic burden on healthcare systems. Such interventions are particularly relevant in primary care where the majority of prescribing of medications takes place. There is currently no single interventional strategy that has proved to be most effective, but there is an argument that a multifaceted approach, which combines a number of techniques within a single intervention, may be likely to be more effective $(6,7)$. To date, a limited number of multifaceted interventions targeting inappropriate prescribing reductions have been evaluated in primary care and report positive findings (8-10). Nonetheless, further evidence on clinical and cost effectiveness is required before widespread adoption in primary care can be recommended. 
The OPTI-SCRIPT (Optimizing Prescribing for Older People in Primary Care: A Cluster Randomised Controlled Trial) study sought to explore these questions for the primary care setting in Ireland (10-13). To this end, a cluster randomized controlled trial (RCT) was conducted to evaluate a multifaceted intervention, incorporating academic detailing with a pharmacist and medicines' review with web-based pharmaceutical treatment algorithms, to reduce potentially inappropriate prescribing among older people (10-13). In the clinical effectiveness analysis the results indicated that, relative to usual care, the intervention was associated with a significant reduction in PIPs overall, largely driven by modifications to the prescribing of proton pump inhibitors $(10,13)$. In addition to clinical effectiveness, any decision regarding the adoption of healthcare interventions in clinical practice should depend upon their expected cost effectiveness (14). The techniques of economic evaluation are concerned with the estimation of such evidence and in this study, we report the results of the economic evaluation conducted to examine the cost effectiveness of the OPTI-SCRIPT intervention.

\section{METHODS}

\section{The OPTI-SCRIPT Cluster RCT}

Full details of the study methods, consisting of a cluster RCT with 21 general practices and 196 patients, are published elsewhere $(11,12)$. The Research Ethics Committee of the Irish College of General Practitioners (ICGP) approved the study. General practices from the Health Research Board (HRB) Centre for Primary Care Research Network were invited to participate by email with a follow-up phone call. Practices were eligible if they had at least 80 patients aged $\geq 70$ years and were based in Dublin. Consenting practices were instructed to randomly select 50 patients aged $\geq 70$ years with capacity to provide informed consent. Prescriptions of these patients were assigned a study ID and sent to the research team where a research pharmacist determined if they had selected PIPs as per the study inclusion criteria. For the purposes of the trial, PIPs were defined according to the criteria in Supplementary Table 1 $(11,12)$. Eligible patients were sent study information packs by the general practice and those wishing to participate returned signed consent forms to the research team. Baseline data were collected prior to random allocation, which was conducted by an independent researcher using minimisation. It was not possible to blind patients or GPs to allocations; however, the outcome 
assessor was blinded. Full details on the characteristics of the study participants are presented in Supplementary Table 2.

The intervention and control treatment alternatives are outlined in Supplementary Table 3. In brief, practices $(n=10)$ and patients $(n=97)$ randomised to the control group received usual care in primary care in addition to a one-off simple patient-level PIPs feedback. Practices $(n=11)$ and patients $(\mathrm{n}=99)$ randomised to the intervention group received a multifaceted intervention, involving academic detailing with a pharmacist on how to conduct a GP-led medicines review with participating patients; medicines reviews supported by web-based pharmaceutical treatment algorithms for GPs which provided evidence-based alternative treatment options to PIP drugs; and tailored patient information leaflets. The RCT was conducted between October 2012 and September 2013. All general practices and 190 (97\%) patients were followed up on intervention completion at approximately 4 to 6 months and at 12 months at the end of the trial follow up. Six patients ( 3 intervention and 3 control) were lost to follow up, while 35 patients (17 intervention and 18 control) did not return the follow up questionnaire.

\section{Economic Evaluation}

The economic evaluation was conducted following the guidelines for health technology assessment for Ireland (15). It consisted of a trial-based analysis with a time horizon of 12 months; that is, the trial follow up period. The perspective of the healthcare provider was adopted with respect to costing and health outcomes were expressed in terms of PIPs and Quality Adjusted Life Years (QALYs). Data on resource use, PIPs and health status, specifically the Euroqol EQ5D-3L instrument (16) were collected via practice note searches of patient charts and patient questionnaires at baseline and at 12 months follow up. Given the length of follow up, neither costs nor outcomes were discounted.

The statistical analysis was conducted on an intention to treat basis, and in accordance with current guidelines for cluster RCTs $(17,18)$. That is, we adopted multilevel statistical techniques which recognise both the clustering and correlation in the cost and effectiveness data. Descriptive statistics were estimated to summarise the variables of interest. The incremental analysis was undertaken using generalised estimating equations (GEE), a flexible multivariate regression framework that explicitly allows for the modelling of normal and nonnormal distributional forms of clustered data (14). Uncertainty in the analysis was addressed 
by estimating $95 \%$ confidence intervals and cost effectiveness acceptability curves (CEACs), which link the probability of cost effectiveness to a range of potential threshold values $(\lambda)$ that health policy makers may be willing to pay per additional unit of health gain $(19,20)$. In addition, a series of sensitivity analyses were conducted. All analysis was undertaken in the Stata 13 statistical software package [StataCorp College Station, Texas, USA].

\section{Cost Analysis}

Three cost components were included in the analysis, all of which were expressed in Euros $(€)$ in 2013 prices. Resource use was captured via a combination of practice note searches and patient questionnaires, and a vector of unit costs (see Table 1) was applied to calculate the costs associated at baseline and follow up. Unit cost estimates for each activity were based on national data sources (listed in Table 1) and, where necessary, were transformed to Euros $(€)$ in 2013 prices using appropriate indices (15).

The first component related to the cost of implementing the intervention in clinical practice. This included a range of resources including the pharmacist and GP time input relating to the review and identification of PIPs, educational materials and consumables, and travel expenses. Data were recoded prospectively by the study research team and the estimated cost was allocated to all patients in the intervention arm (see Table 1 for further details). In sensitivity analysis, we explored the implications of increasing the intervention cost per patient by $100 \%$.

Second, the costs relating to PIPs were estimated for the intervention and control groups at baseline and at 12 months follow up. Prescriptions for all patients at each time point were sent to the research team where a research pharmacist identified PIPs based on the predefined criteria specified by the clinical research team. Data on the unit cost per prescription were obtained from the primary care reimbursement service (PCRS) for Ireland and applied to estimate the cost of PIPs per patient in both treatment arms at baseline and follow up. Notably, as data on PIPs referred to the specific data collection point at baseline and follow up, a number of assumptions were made to allow for the calculation of the total costs of PIPs over the time horizon of the trial of 12 months follow up. For the base-case analysis, it was assumed that the PIPs observed at 12 months follow up were applicable to the entire 12 month follow up period. In sensitivity analysis, a number of alternative scenarios were explored. First, it was assumed that the PIPs observed at baseline were applicable to the first 6 months and that the PIPs 
observed at follow up were applicable to the final 6 months of follow up. Second, it was assumed that the PIPs observed at baseline were applicable to the first 9 months and the PIPs observed at follow up were applicable to the final 3 months of follow up. In addition, to reflect a change in pricing policy which came into effect after the trial was completed, we conducted a sensitivity analysis to explore the impact of reducing the costs of medications by $50 \%$. Finally, to address a concern that including the cost of PIPs in the numerator and the number of PIPs in the denominator may represent double counting in the incremental cost per PIP avoided analysis, we present an analysis with the cost of PIPs excluded.

Third, the costs relating to the use of primary and secondary healthcare services for the 12 months prior to baseline and for the 12 months follow up period were estimated for patients in both treatment arms. This included the costs of GP and practice nurse consultations, outpatient clinic visits, A\&E clinic visits, and hospital day-case and inpatient admissions.

For the incremental cost analysis, a healthcare provider perspective was adopted and the three cost components were combined to generate a total cost variable per patient. Estimation of incremental costs at follow up was undertaken using a GEE regression model controlling for the treatment arm, baseline cost, age, gender, baseline number of PIPs, number of GPs per practice, and practice location. To account for the non-normal nature of the cost data, a Gamma variance function were adopted and to account for the hierarchical nature of data, an exchangeable correlation structure was used (14). In sensitivity analysis, a GEE regression model controlling only for the treatment arm was estimated.

\section{Effectiveness Analysis}

Health outcomes were expressed in terms of PIPs and QALYs. Complete data were available with respect to the number of PIPs in both treatment arms at baseline and follow up. Estimation of incremental number of PIPs at follow up was undertaken using a GEE regression model, assuming a Gaussian variance function, an identity link function and an exchangeable correlation structure, and controlling for the treatment arm, age, gender, baseline number of PIPs, number of GPs per practice, practice location, and clustering. In sensitivity analysis, a GEE regression model controlling only for the treatment arm was estimated. 
Health outcomes were also expressed in terms of QALYs gained which were calculated based on patient responses at baseline and follow up to the EuroQol EQ5D-3L instrument (16). The EQ5D responses were transformed using an algorithm into a single health state index score, based on values elicited via the time trade-off approach for the UK population (16). EQ5D scores at baseline and 12 months follow up were used to calculate patient-specific QALYs gained over 12 months using the area under the curve method (14). Estimation of incremental QALYs was undertaken using a GEE regression model, assuming a Gaussian variance function, an identity link function and an exchangeable correlation structure, and controlling for treatment arm, baseline EQ5D score, age, gender, baseline number of PIPs, number of GPs per practice, practice location, and clustering. In sensitivity analysis, a GEE regression model controlling for the treatment arm only was estimated.

Notably, missing data proved to be an issue with respect to the EQ5D data and the resultant QALYs estimates. At baseline, data availability was $46 \%$ for the intervention and $65 \%$ for the control. At follow up, these values were $41 \%$ and $65 \%$, respectively. As there was no clear evidence of systematic bias with respect to the missing data, in sensitivity analysis, multiple imputation was undertaken to estimate missing values for EQ5D data to explore the implications for the incremental QALYs results. The imputation models for EQ5D data included age, gender, practice, and treatment arm (at follow up only) and were estimated using predictive mean matching, based on $\mathrm{M}=5$ imputed data sets. The analysis was undertaken using the MI estimate mixed and MI predict commands in Stata 13, which estimate the regression model on each of the imputed data sets and apply Rubin's rules to generate the coefficients of interest (21).

\section{Cost Effectiveness Analysis}

To undertake economic evaluation alongside cluster RCTs, techniques which recognise the clustering and correlation in the cost and effectiveness data must be adopted $(18,20)$. Separate multilevel regression models, controlling for treatment arm, clustering, and a range of other covariates, were used to estimate the incremental costs, PIPs and QALYs for the intervention relative to the control. The uncertainty surrounding these point estimates was examined using CEACs, which report the probability of an intervention being cost effective, relative to a comparator, for a range of potential threshold values per unit of health gain (19). In the Irish context, it is recommended that results be presented for cost effectiveness thresholds of up to 
$€ 45,000$ per QALY gained (15). As no such recommendation exist for potentially inappropriate prescribing related outcomes, results are presented for a range of values up to $€ 4,500$ per PIP avoided. Notably, we explicitly assume that the threshold values adopted, which are typically assumed to apply over a lifetime time horizon, are applicable to the 12 month time horizon of our evaluation.

\section{RESULTS}

Descriptive statistics for resource use, costs and health outcomes at baseline and follow up are presented in Table 2. The total cost of implementing the intervention was $€ 5,824$ giving a mean cost per patient estimate of $€ 58$. Summary data for the proportion of patients with each category of PIP at baseline and follow up are reported in Table 3. Notably, prescriptions of proton pump inhibitors constituted the largest single class of PIP identified in the intervention and control arms at baseline and follow up.

The results from the incremental analyses are presented in Table 4 . In terms of total costs at 12 months follow up, the estimated mean cost per patient was $€ 3075$ (95\% Confidence Interval (CI): 2704, 3446) for the intervention group and $€ 2668$ (95\% CI: 2297, 3040) for the control group. The intervention was associated, on average, with an increase in cost of $€ 407$ (95\% CIs: $-357,1170)$ per patient compared to the control.

With respect to health outcomes, the mean number of PIPs at 12 months follow up was estimated at 0.627 (95\% CI: 0.588, 0.666) for the intervention group and 1.006 (95\% CI: 0.967 , $1.045)$ for the control group. The intervention was associated with a mean reduction of 0.379 (95\% CI: -0.666, -0.092) PIPs relative to the control. Mean QALYs gained per patient at 12 months was estimated to be $0.671(95 \% \mathrm{CI}: 0.625,0.716)$ in the intervention group and 0.657 (95\% CI: $0.612,0.703)$ in the control group. The intervention was associated, on average, with an increase of 0.013 (95\% CIs: $-0.016,0.042)$ in QALYs gained.

These estimates translated into incremental cost effectiveness ratios (ICERs) of $€ 30,535$ (95\% CI: -334846, 289498) per QALY gained and $€ 1,269$ (95\% CI: -1400, 6302) per PIP avoided. The probabilistic results are summarised in Table 4 and Supplementary Figures 1 and 2 and indicate that the probability of the intervention being cost effective was at least 0.845 at 
threshold values of $€ 2,500$ per PIP avoided and higher. Notably, the probability of the intervention being cost effective was only 0.602 at a threshold value of $€ 45,000$ per QALY gained.

The results from the sensitivity analyses are presented in Supplementary Tables 4-10. These broadly reflect the results from the base-case analysis, with the intervention consistently estimated to be more costly and more effective than the control. Notably, the imputed cost utility analysis reported higher estimates for the probability of the intervention being cost effective relative to the control. Additional results from univariate analysis of resource costs are presented in Supplementary Table 11 and indicate that only the costs of PIPs differed significantly across treatment arms at follow up.

\section{DISCUSSION}

This study examined the cost effectiveness of the OPTI-SCRIPT intervention, which has been shown to be effective in reducing potentially inappropriate prescribing relative to usual primary care in Ireland (10-13). The results indicate that, on average, the intervention was more costly and more effective, in terms of PIPs avoided and QALYs gained, compared to the control. Notably however, while there was evidence of a statistically significant reduction in PIPs, there was no such evidence for the difference in mean costs or the difference in mean QALYs. That is, the observed reduction in PIPs did not translate into statistically significant improvements in QALYs or statistically significant reductions in healthcare costs. Indeed, this finding reflects the ambiguity that exists in the literature with respect to the longer term implications of PIPs. For example, recent prospective cohort studies have reported that PIPs were associated with increased healthcare utilisation, increased ADEs and diminished quality of life $(22,23)$; whereas other studies have reported the magnitude of these effects to be modest $(2,3,4)$.

This raises the question of whether the lack of evidence with respect to QALYs reflects an absence of a clinically significant treatment effect or whether it reflects a lack of sensitivity in the EQ5D instrument, or indeed how we have employed it in our analysis, to detect a clinically meaningful improvement in health status for the older patient population under consideration. 
It may also be the case that our analysis, which was limited to a time horizon of the 12 months, was too short to capture the longer terms implications of reducing PIPs. Indeed, a common criticism of the evaluation literature for prescribing interventions is that sample sizes may be too small and follow-up periods too short to detect differences in patient reported outcomes (24). Finally, a further explanation may be that our findings related mainly to proton pump inhibitor prescriptions and reductions in such prescriptions may not significantly impact upon self-rated health status.

Our results also indicated that the reduction in PIPs did not lead to a statistically significant difference in mean healthcare costs between the intervention and the control. Indeed, and although not statistically significant, when intervention costs, PIP costs, and other healthcare service costs over the 12 month follow up period were combined, the intervention was, on average, more costly than the control. That said, there was no evidence of statistically significant differences in the costs of individual primary or secondary care services between the intervention and control over the course of the trial.

These issues notwithstanding, in circumstances where an intervention is, on average, more costly and more effective than a control comparator, it will only be judged cost effective if its ICER falls below the threshold value that policy makers would be willing to pay for an additional unit of health gain (14). The ICER from the cost utility analysis of $€ 30,535$ per QALY gained is likely to be viewed favourably in the Irish context, and in other similar jurisdictions, where a threshold value in the region of $€ 45,000$ per QALY has been proposed (15). Nonetheless, the uncertainty surrounding this ICER point estimate must be also taken into account. In this case, the probabilistic results indicate the likelihood of the intervention being cost effective to be 0.602 at a threshold value of $€ 45,000$ per QALY. Such a level of uncertainty is unlikely to be deemed sufficient to justify adoption in clinical practice. Notably, the response rate to the EQ5D questionnaire had a direct impact of this finding as this probability estimate increased to 0.764 when the missing EQ5D score data was imputed and the cost utility analysis was re-estimated.

The results from the cost effectiveness analysis provide additional evidence. Importantly, while there is no formal threshold value per PIP avoided for Ireland or elsewhere, we present results 
for a set of potential threshold values for illustrative purposes. The robust findings for the statistically significant reduction in PIPs associated with the intervention are reflected in the cost effectiveness probability estimates, which increase as the threshold value per PIP avoided increases. For example, if decision makers were willing to pay at least $€ 2,500$ to avoid a PIP, there is a probability of 0.845 or higher of the intervention being cost effective. That said, whether or not this proves to be an appropriate range of threshold values in this context is open to debate. Taken together, the ultimate determination of the cost effectiveness of the OPTISCRIPT intervention will depend on the relevant policy maker's threshold value per PIP avoided and QALY gained, as well as their attitude towards uncertainty.

This study adds to the limited literature of interventions targeting reductions in potentially inappropriate prescribing in primary care. Of those interventions that have been evaluated, single interventions such as computerised decision support systems (CDSSs), educational interventions, and multidisciplinary teams have produced inconsistent effects $(2,24,25)$. More recently, two separate RCTs have found that multifaceted interventions are effective $(8,9)$. Rognstad et al (9) found that peer academic detailing, delivered at continuing medical education (CME) meetings, with mailed prescriber feedback, produced a 10\% (95\% CI 5.9 to 15.0) reduction in PIP. Similarly, Bregnhoj et al (8) found that interactive educational meetings and feedback resulted in a 5 point (95\% CI: 2.6 to 7.3 ) improvement in the medication appropriateness index (MAI) score. Differences in effect sizes reported between these studies and the OPTI-SCRIPT findings may arise from a number of factors including differences in the criteria used to assess PIPs, the duration of follow-up and the patients included and the context of care $(10,13)$.

The study was subject to a number of limitations. First, the limitations associated with the RCT $(11,12)$ on which the analysis was based are also applicable to the economic evaluation. There were a number of issues with respect to the cost analysis. In the case of costing PIPs, given data issues, we made the explicit assumption that all PIPs represented inappropriate cases and there were no cases in which a PIP would have been substituted for an alternative medication. In addition, we made a series of assumptions to cost PIPs over the 12 month trial follow up period. Furthermore, while the cost analysis was conducted from the healthcare service perspective and included a broad range of resource use activities, certain resource items were not captured. For example, the costs of community care services, private patient costs such as private health insurance premiums, and broader costs to society such as productivity losses 
were not captured. Nonetheless, there is little evidence to suggest that the inclusion of such resource categories would have significantly changed the results. Finally, the process of conducting cost analysis in Ireland is compromised by the lack of nationally available unit cost data. In estimating unit costs for individual resource activities, we endeavoured at all times to be conservative in any assumptions adopted. It should also be noted that we adopt 2013 prices for the analysis and medical inflation has fallen in the period since the trial was conducted. To examine the uncertainty surrounding the cost analysis, a series of sensitivity analysis were conducted, the results from which did not differ fundamentally from those of the base-case analysis.

There were also a number of limitations in relation to the health outcome analysis. In particular, the available PIP data only reflected medications that were prescribed and did not capture whether or not the medication was subsequently dispensed and taken by the patient. With respect to the estimation of QALYs, given the lack of Irish utility data for the EQ5D-3L instrument, the equivalent UK algorithm was adopted and assumed to be generalisable to the older Irish population. Furthermore, the patient response rate for the EQ5D-3L questionnaire was disappointing and was reflected in the cost utility analysis results. In sensitivity analysis, after careful consideration of missing data patterns, we proceeded with the assumption that the data were missing at random and multiple imputation was undertaken to impute missing values. The imputation process adopted is subject to criticism as the variables included in the imputation models were pragmatically chosen by the study team and the values for costs and EQ5D scores were imputed independently (18). Nonetheless, the imputed results provide useful additional information on the potential cost effectiveness of the intervention.

Finally, in the case of chronic disease, a lifetime time horizon for analysis is encouraged as interventions may have long term implications which occur beyond the end of trial follow up (14). Additional follow up of the trial sample should be conducted to explore the longer term implications of the results presented here. 


\section{CONCLUSION}

This study presents cost effectiveness evidence for the OPTI-SCRIPT intervention in reducing potentially inappropriate prescribing in primary care in Ireland. While we report that the OPTISCRIPT intervention was effective in reducing PIPs, uncertainty exists with respect to its cost effectiveness.There was strong evidence that the intervention was cost-effective if decision makers were willing to pay at least $€ 1,269$ per PIP avoided; however the evidence was much less compelling when effectiveness was measured in terms of QALYs gained. Further studies are required to explore the longer term health and economic implications of interventions targeting potentially inappropriate prescribing in primary care.

\section{CONFLICTS OF INTEREST}

All authors have no conflict of interest to declare. 


\section{References}

1. Kaufmann C, Tremp R, Hersberger K, Lampert M. Inappropriate prescribing: a systematic overview of published assessment tools. Eur J Clin Pharmacol. 2014;70(1):1-11.

2 Spinewine A, Schmader K, Barber N, Hughes C, Lapane K, Swine C, et al. Appropriate prescribing in elderly people: how well can it be measured and optimised? Lancet. 2007;370:173- 84.

3 Hamilton HJ, Gallagher PF, O'Mahony D. Inappropriate prescribing and adverse drug events in older people. BMC Geriatr. 2009;9:5.

4 Cahir C, Fahey T, Teeling M, Teljeur C, Feely J, Bennett K. Potentially inappropriate prescribing and cost outcomes for older people: A national population study. Br J Clin Pharmacol. 2010;69(5):54352.

5 Cahir C, Moriarty F, Teljeur C, Fahey T, Bennett K. Potentially Inappropriate Prescribing and Vulnerability and Hospitalization in Older Community-Dwelling Patients. Annals of Pharmacotherapy. 2014;48(12):1546-54.

6 Majumdar SR, Soumerai SB. Why most interventions to improve physician prescribing do not seem to work. CMAJ. 2003;169(1):30-1.

7 Grimshaw JM, Shirran L, Thomas R, Mowatt G, Fraser C, Bero L, et al. Changing Provider Behavior: An Overview of Systematic Reviews of Interventions. Medical Care. 2001;39(8):II2-II45.

8 Bregnhoj L, Thirstrup S, Kristensen M, Bjerrum L, Sonne J. Combined intervention programme reduces inappropriate prescribing in elderly patients exposed to polypharmacy in primary care. Eur J Clin Pharmacol. 2009;65(2):199-207.

9 Rognstad S, Brekke M, Fetveit A, Dalen I, Straand J. Prescription peer academic detailing to reduce inappropriate prescribing for older patients: a cluster randomised controlled trial. $\mathrm{Br} J$ Gen Pract. 2013;63(613):e554-e62.

10 Clyne B, Smith SM, Hughes CM, Boland F, Bradley MC, Cooper JA, et al. Effectiveness of a Multifaceted Intervention for Potentially Inappropriate Prescribing in Older Patients in Primary Care: A Cluster-Randomized Controlled Trial (OPTI-SCRIPT Study). Ann Fam Med. 2015;13(6):545-53.

11 Clyne B, Bradley M, Hughes C, Clear D, McDonnell R, Williams D, et al. Addressing potentially inappropriate prescribing in older patients: development and pilot study of an intervention in primary care (the OPTI-SCRIPT study). BMC Health Serv Res. 2013;13(1):307.

12 Clyne B, Bradley MC, Smith SM, Hughes CM, Motterlini N, Clear D, et al. Effectiveness of medicines review with web-based pharmaceutical treatment algorithms in reducing potentially inappropriate prescribing in older people in primary care: a cluster randomized trial (OPTI-SCRIPT study protocol). Trials. 2013;14(1):72.

13. Clyne B, Smith S, Hughes C, Boland F, Cooper J, Fahey T, et al. Sustained effectiveness of a multifaceted intervention to reduce potentially inappropriate prescribing in older patients in primary care (OPTI-SCRIPT study). Implementation Science. 2016;11(1):79.

14 Drummond MF, Sculpher MJ, Torrance GW, O'Brien BJ, Stoddart GL. Methods for the Economic Evaluation of Health Care Programmes. New York: Oxford University Press; 2015.

15 Health Information and Quality Authority. Guidelines for the Economic Evaluation of Health Technologies in Ireland. Dublin: Health Information and Quality Authority, 2010.

16 The EuroQol Group. EuroQol--a new facility for the measurement of health-related quality of life. Health Policy. 1990;16(3):199-208.

17 Campbell MK, Elbourne DR, Altman DG. CONSORT statement: extension to cluster randomised trials. BMJ. 2004;328(7441):702-8.

18 Gomes M, Ng ES, Grieve R, Nixon R, Carpenter J, Thompson SG. Developing appropriate methods for cost-effectiveness analysis of cluster randomized trials. Medical decision making : an international journal of the Society for Medical Decision Making. 2012;32(2):350-61.

19 Fenwick E, Byford S. A guide to cost-effectiveness acceptability curves. The British journal of psychiatry : the journal of mental science. 2005;187:106-8.

$20 \mathrm{Ng}$ ES, Grieve R, J C. Two-stage non-parametric bootstrap sampling with shrinkage correction for clustered data. Stata J. 2013;15:141-64. 
21 Rubin D. Multiple Imputation for Nonresponse in Surveys. Chichester: Wiley; 1987.

22 Moriarty F, Bennett K, Cahir C, Kenny RA, Fahey T.Potentially inappropriate prescribing according to STOPP and START and adverse outcomes in community-dwelling older people: a prospective cohort study. Br J Clin Pharmacol. 2016 Sep;82(3):849-57. doi: 10.1111/bcp.12995.

23 Wallace E, McDowell R, Bennett K, Fahey T, Smith SM.Impact of Potentially Inappropriate Prescribing on Adverse Drug Events, Health Related Quality of Life and Emergency Hospital Attendance in Older People Attending General Practice: A Prospective Cohort Study. J Gerontol A Biol Sci Med Sci. 2017 Feb;72(2):271-277. doi: 10.1093/gerona/glw140.

24 Yourman L, Concato J, Agostini JV. Use of computer decision support interventions to improve medication prescribing in older adults: A systematic review. Am J Geriatr Pharmacother. 2008;6(2):119-29.

25 Kaur S, Mitchell G, Vitetta L, Roberts MS. Interventions that can reduce inappropriate prescribing in the elderly: a systematic review. Drugs Aging. 2009;26(12):1013-28. 
Table 1 - Categories of resource use and unit cost estimates in $2013(€)$ prices

\begin{tabular}{|c|c|c|c|}
\hline Resource item & Activity & $\begin{array}{c}\text { Unit cost } \\
€\end{array}$ & Source \\
\hline \multicolumn{4}{|l|}{ OPTI-SCRIPT intervention } \\
\hline General Practitioner( GP) review consultation & Per Patient & 50 & Study Accounts \\
\hline Pharmacist Academic Detailing & Per Patient & 4 & Study Accounts \\
\hline Travel Expenses & Per Patient & 1.50 & Study Accounts \\
\hline Materials and Consumables & Per Patient & 2.50 & Study Accounts \\
\hline \multicolumn{4}{|l|}{ Healthcare resources } \\
\hline General Practitioner(GP) Visits & Per Consultation & 50 & ORC \\
\hline $\begin{array}{l}\text { Practice Nurse Visits } \\
\text { Prof }\end{array}$ & Per Consultation & 12 & ORC \\
\hline Outpatient Clinic Visits & Per Consultation & 142 & DOHC \\
\hline Day Case Admission & Per Day & 671 & DOHC \\
\hline Inpatient Admission & Per Night & 777 & DOHC \\
\hline Accident and Emergency (A\&E) Visits & Per Consultation & 270 & DOHC \\
\hline \multicolumn{4}{|l|}{ Potentially inappropriate prescriptions } \\
\hline Proton Pump Inhibitor (PPI) & Per Pack & EUR 6.90 - EUR 13.72 & PCRS \\
\hline Nonsteroidal anti-inflammatory drug (NSAID) & Per Pack & EUR5.04 - EUR89.39 & PCRS \\
\hline Long-term benzodiazepines (Long acting benzo) & Per Pack & EUR 1.10- EUR 8.07 & PCRS \\
\hline Short-acting benzodiazepines( BenzoSA) & Per Pack & EUR 2.12 - EUR 19.15 & PCRS \\
\hline Tricyclic Anti-depressant (TCA) & Per Pack & EUR 3.70- EUR 14.80 & PCRS \\
\hline Aspirin & Per Pack & EUR 1.03- EUR 36.5 & PCRS \\
\hline Theophylline & Per Pack & EUR 2.30 - EUR 8.35 & PCRS \\
\hline Antihistamine & Per Pack & EUR 1.91 & PCRS \\
\hline Bladder antimuscarinic drugs (BA) & Per Pack & EUR 20.69- EUR 34.26 & PCRS \\
\hline $\begin{array}{l}\text { Digoxin } \\
\text { Dige }\end{array}$ & Per Pack & EUR 8.48- EUR 9.04 & PCRS \\
\hline Thiazide diuretic (TD) & Per Pack & EUR 2.34- EUR 33.59 & PCRS \\
\hline Glibenclamide & Per Pack & EUR 6.20 & PCRS \\
\hline Prochlorperazine or metoclopramide $(\mathrm{P} / \mathrm{M})$ & Per Pack & EUR 4.35-EUR11.98 & PCRS \\
\hline Calcium channel blockers (CCB) & Per Pack & EUR 5.85- EUR9.46 & PCRS \\
\hline Long-term corticosteroids (LT steroid) & Per Pack & EUR 6.21- EUR 19.09 & PCRS \\
\hline Chronic obstructive pulmonary disease (COPD) steroid & Per Pack & EUR 6.40- EUR 21.60 & PCRS \\
\hline Osteoarthritis $(\mathrm{OA})$ steroid & Per Pack & EUR 6.40- EUR 21.60 & PCRS \\
\hline
\end{tabular}

ORC - Office of the Revenue Commissioner, Dublin, Ireland.

DOHC - Casemix Unit, Department of Health and Children, Dublin, Ireland

PCRS - Primary Care Reimbursement Service, Health Service Executive, Dublin, Ireland 
Table 2 - Resource Use, costs and health outcomes at baseline and 12 months follow up

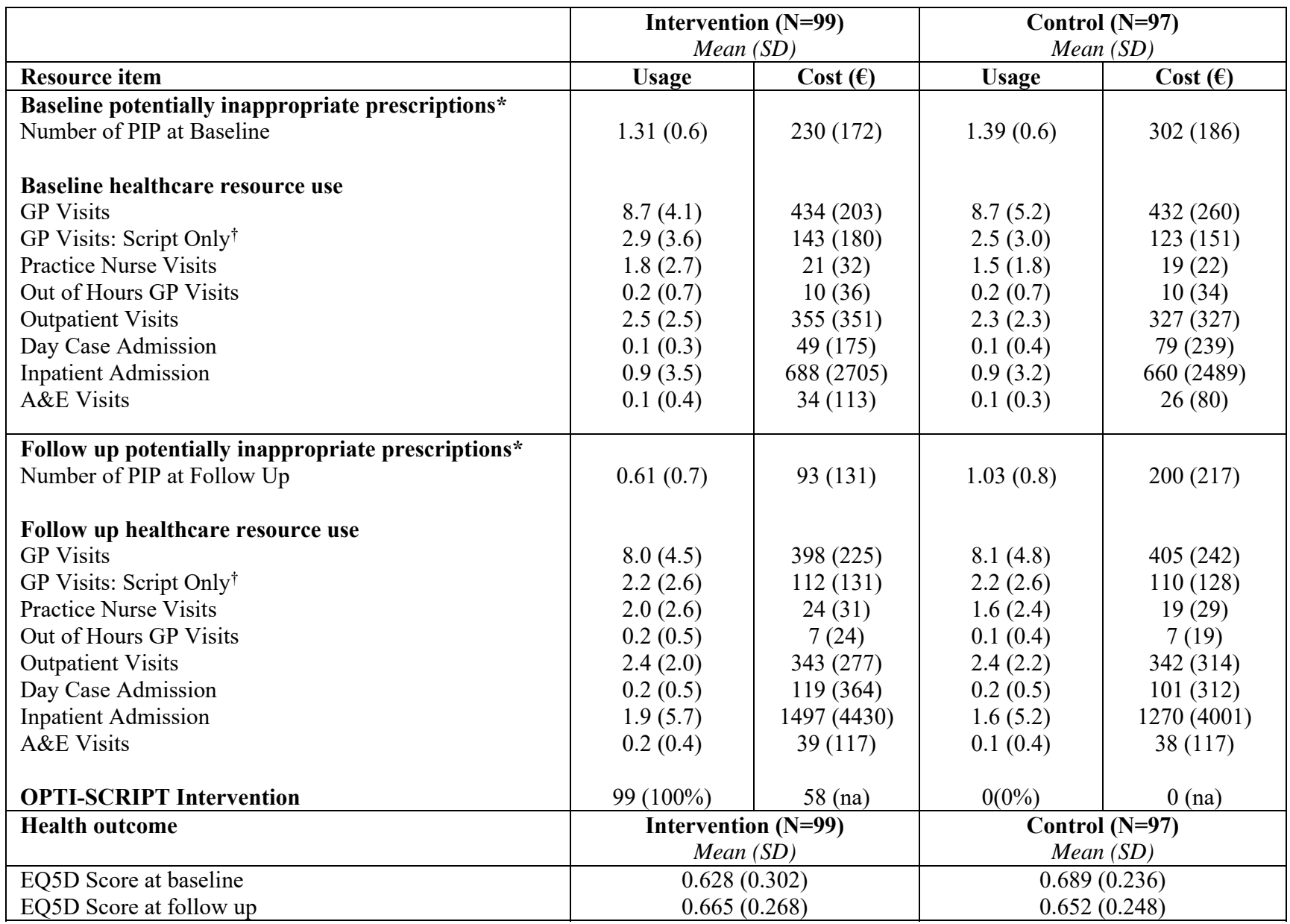

Completeness of data at baseline: Intervention - 96/99 (97\%) for primary and secondary care utilisation, $99 / 99$ (100\%) for PIPs, 45/99 (46\%) for EQ5D. Control: 93/97 (96\%) for primary and secondary care utilisation, 97/97 (100\%) for PIPs, 63/97 $(65 \%)$ for EQ5D.

Completeness of data at follow up: Intervention - 96/99 (97\%) for primary and secondary care utilisation, 99/99 (100\%) for PIPs, 41/99 (41\%) for EQ5D, 37/99 (37\%) for QALY and 95/99 (96\%) for Total Cost. Control: 93/97 (96\%) for primary and secondary care utilisation, $97 / 97$ (100\%) for PIPs, 63/97 (65\%) for EQ5D, 54/97 (56\%) for QALY and 93/97 (96\%) for Total Cost.

* Potentially inappropriate prescriptions are medicines whose potential harms may outweigh their benefits. The PIPs inclusion criteria are described in Supplementary Table 1. Patient repeat medications were reviewed by a research pharmacist to confirm if patients had PIP as per this list.

$\dagger$ For repeat prescriptions, GPs may pre authorise for patients to have a script issued, without having a GP consultation. 
Table 3 - Potentially inappropriate prescriptions at baseline and 12 months follow up

\begin{tabular}{|l|c|c|c|c|}
\hline Potentially inappropriate prescriptions (PIP)* & \multicolumn{2}{|c|}{ Intervention (N=99) } & \multicolumn{2}{c|}{$\begin{array}{c}\text { Control (N=97) } \\
\%\end{array}$} \\
\hline & Baseline & Follow Up & Baseline & Follow Up \\
\hline Proton pump inhibitor (PPI) & $53 \%$ & $26 \%$ & $68 \%$ & $43 \%$ \\
Nonsteroidal anti-inflammatory drug (NSAID) & $21 \%$ & $3 \%$ & $17 \%$ & $8 \%$ \\
Long-term benzodiazepines (Long acting benzo) & $14 \%$ & $8 \%$ & $8 \%$ & $9 \%$ \\
Short-acting benzodiazepines(BenzoSA) & $0 \%$ & $0 \%$ & $0 \%$ & $0 \%$ \\
Tricyclic Anti-depressant (TCA) & $1 \%$ & $1 \%$ & $5 \%$ & $3 \%$ \\
Aspirin & $3 \%$ & $5 \%$ & $1 \%$ & $2 \%$ \\
Theophylline & $0 \%$ & $0 \%$ & $0 \%$ & $0 \%$ \\
Antihistamine & $4 \%$ & $1 \%$ & $2 \%$ & $2 \%$ \\
Bladder antimuscarinic drugs (BA) & $1 \%$ & $2 \%$ & $9 \%$ & $6 \%$ \\
Digoxin & $1 \%$ & $0 \%$ & $3 \%$ & $3 \%$ \\
Thiazide diuretic (TD) & $3 \%$ & $1 \%$ & $2 \%$ & $2 \%$ \\
Glibenclamide & $0 \%$ & $0 \%$ & $0 \%$ & $0 \%$ \\
Prochlorperazine or metoclopramide (P/M) & $0 \%$ & $0 \%$ & $0 \%$ & $0 \%$ \\
Calcium channel blockers (CCB) & $0 \%$ & $2 \%$ & $3 \%$ & $2 \%$ \\
Long-term corticosteroids (LT_steroid) & $0 \%$ & $0 \%$ & $0 \%$ & $1 \%$ \\
Chronic obstructive pulmonary disease (COPD) steroid & $0 \%$ & $0 \%$ & $0 \%$ & $0 \%$ \\
Osteoarthritis (OA) steroid & $9 \%$ & $3 \%$ & $4 \%$ & $2 \%$ \\
Therapeutic Duplication & $19 \%$ & $3 \%$ & $14 \%$ & $9 \%$ \\
\hline
\end{tabular}

* Potentially inappropriate prescriptions are medicines whose potential harms may outweigh their benefits. The PIPs inclusion criteria are described in Supplementary Table 1. Patient repeat medications were reviewed by a research pharmacist to confirm if patients had PIP as per this list. 
Table 4 - Incremental cost effectiveness analysis and cost utility analysis results

\begin{tabular}{|c|c|c|c|c|}
\hline & \multicolumn{4}{|c|}{$\begin{array}{l}\text { INCREMENTAL ANALYSIS } \\
\text { (Intervention versus Control) }\end{array}$} \\
\hline Cost Analysis & \multicolumn{2}{|c|}{ Intervention } & \multicolumn{2}{|c|}{ Control } \\
\hline $\begin{array}{l}\text { Healthcare Costs } \\
\text { (95\% CIs) }\end{array}$ & \multicolumn{2}{|c|}{$\begin{array}{c}3075 \\
(2704,3446)\end{array}$} & \multicolumn{2}{|c|}{$\begin{array}{c}2668 \\
(2297,3040)\end{array}$} \\
\hline $\begin{array}{l}\text { Difference in Healthcare Cost } \\
(95 \% \text { CIs })\end{array}$ & \multicolumn{4}{|c|}{$\begin{array}{c}407 \\
(-357,1170)\end{array}$} \\
\hline & \multicolumn{4}{|c|}{$\begin{array}{c}\text { INCREMENTAL ANALYSIS } \\
\text { (Intervention versus Control) }\end{array}$} \\
\hline Effectiveness Analysis & \multicolumn{2}{|c|}{ Intervention } & \multicolumn{2}{|c|}{ Control } \\
\hline $\begin{array}{l}\text { Number of PIPs } \\
(95 \% \text { CIs })\end{array}$ & \multicolumn{2}{|c|}{$\begin{array}{c}0.627 \\
(0.588,0.666)\end{array}$} & \multicolumn{2}{|c|}{$\begin{array}{c}1.006 \\
(0.967,1.045)\end{array}$} \\
\hline $\begin{array}{l}\text { Difference in Number of PIPs } \\
(95 \% \mathrm{CI})\end{array}$ & \multicolumn{4}{|c|}{$\begin{array}{c}-0.379 \\
(-0.666,-0.092)\end{array}$} \\
\hline & \multicolumn{4}{|c|}{$\begin{array}{l}\text { INCREMENTAL ANALYSIS } \\
\text { (Intervention versus Control) }\end{array}$} \\
\hline Effectiveness Analysis & \multicolumn{2}{|c|}{ Intervention } & \multicolumn{2}{|c|}{ Control } \\
\hline $\begin{array}{l}\text { QALYs } \\
(95 \% \text { CIs })\end{array}$ & \multicolumn{2}{|c|}{$\begin{array}{c}0.671 \\
(0.625,0.716)\end{array}$} & \multicolumn{2}{|c|}{$\begin{array}{c}0.657 \\
(0.612,0.703)\end{array}$} \\
\hline Difference in QALYs $(95 \% \mathrm{CI})$ & \multicolumn{4}{|c|}{$\begin{array}{c}0.013 \\
(-0.016,0.042) \\
\end{array}$} \\
\hline & \multicolumn{4}{|c|}{$\begin{array}{c}\text { INCREMENTAL ANALYSIS } \\
\text { (Intervention versus Control) }\end{array}$} \\
\hline $\begin{array}{l}\text { ICER per PIP avoided }(€) \\
(95 \% \text { CIs })\end{array}$ & \multicolumn{4}{|c|}{$\begin{array}{c}1269 \\
(-1400,6302)\end{array}$} \\
\hline $\begin{array}{l}\text { ICER per QALYs gained }(€) \\
(95 \% \text { CIs })\end{array}$ & \multicolumn{4}{|c|}{$\begin{array}{c}30535 \\
(-334846,289498)\end{array}$} \\
\hline $\begin{array}{l}\text { Probability that the Intervention is Cost } \\
\text { Effective }\end{array}$ & \multicolumn{2}{|c|}{ PIP Avoided } & \multicolumn{2}{|c|}{ QALY Gained } \\
\hline & $\begin{array}{l}\text { Threshold } \\
\text { Value }(\lambda)\end{array}$ & $\mathbf{P}[\mathbf{C E}]$ & $\begin{array}{l}\text { Threshold } \\
\text { Value }(\lambda)\end{array}$ & $\mathbf{P}[\mathbf{C E}]$ \\
\hline & $\lambda=€ \mathbf{0}$ & 0.143 & $\lambda=€ \mathbf{0}$ & 0.138 \\
\hline & $\lambda=€ 500$ & 0.278 & $\lambda=€ 5,000$ & 0.184 \\
\hline & $\lambda=€ 1,500$ & 0.654 & $\lambda=€ 15,000$ & 0.315 \\
\hline & $\lambda=€ \mathbf{2 , 5 0 0}$ & 0.845 & $\lambda=€ 25,000$ & 0.454 \\
\hline & $\lambda=€ \mathbf{3 , 5 0 0}$ & 0.926 & $\lambda=€ 35,000$ & 0.534 \\
\hline & $\lambda=€ 4,500$ & 0.951 & $\lambda=€ 45,000$ & 0.602 \\
\hline
\end{tabular}

Completeness of Data: Intervention -99/99 (100\%) for PIPs, $37 / 99$ (37\%) for QALY and 95/99 (96\%) for Total Cost. Control: $97 / 97$ (100\%) for PIPs, 54/97 (56\%) for QALY and 93/97 (96\%) for Total Cost.

Incremental Cost Analysis: Estimated by a generalised estimating equation regression model, assuming a Gamma variance function, an identity link function and an exchangeable correlation structure, controlling for treatment arm, age, gender, baseline number of PIP drugs, baseline cost, number of GPs and practice location.

Incremental PIP Analysis: Estimated by a generalised estimating equation regression model, assuming a Gaussian variance function, an identity link function and an exchangeable correlation structure, controlling for treatment arm, age, gender, baseline number of PIP drugs, number of GPs and practice location.

Incremental QALY Analysis: Estimated by a generalised estimating equation regression model, assuming a Gaussian variance function, an identity link function and an exchangeable correlation structure, controlling for treatment arm, age, gender, baseline number of PIP drugs, baseline eq5d score, number of GPs and practice location. 